\title{
Anatomical description of the sciatic nerve block at the subgluteal region in a neonatal cadaver population
}

Adrienne A Acar ${ }^{1}$

Adrian T Bosenberg ${ }^{2}$

Albert-Neels van Schoor ${ }^{1, *}$

${ }^{1}$ Department of Anatomy, Faculty of Health Sciences, University of Pretoria, Pretoria, South Africa

${ }^{2}$ Director Regional Anesthesia, Department Anesthesiology and Pain Management, Seattle Children's Hospital, Seattle, USA.

${ }^{*}$ Corresponding author:

Prof. A van Schoor

Department of Anatomy, Faculty of Health Sciences, University of Pretoria, Private Bag x323, Arcadia, 0007, South Africa

Email: albert.vanschoor@up.ac.za

What is already known

- Sciatic nerve blocks provide intraoperative and prolonged postoperative pain management.

- Current techniques rely on identifiable landmarks or ultrasound guidance developed from extensive anatomical studies performed on adults.

What this article adds

- We verify the ideal position for sciatic nerve blocks in neonates from anatomical data.

- The ideal needle insertion point is approximately halfway between the greater trochanter and the tip of the coccyx. 


\section{Abstract}

Introduction: Sciatic nerve blocks provide intra-operative and prolonged postoperative pain management after major knee or foot surgery. Effective placement of the needle requires sound anatomical knowledge. Anatomical studies for children are uncommon, which limits the use of sciatic nerve blocks. Accurate anatomical information may be used to place sciatic nerve blocks when ultrasound guidance is unavailable. We describe the location of the sciatic nerve at the gluteal level in a neonatal population using clearly defined anatomical landmarks.

Methods: We identified the sciatic nerve in the gluteal and thigh region of 20 neonatal cadavers. The skin covering the gluteal and thigh region were reflected laterally and the underlying structures and muscles were identified. We located the sciatic nerve and measured the distance from the nerve to the greater trochanter of the femur and to the tip of the coccyx with a mechanical dial caliper. The total distance between the two landmarks were then recorded.

Results: A paired t-test revealed no differences between the left and right sides of the sample. We combined measurements from both sides to form a sample size $n=40$. The sciatic nerve was $14.9 \mathrm{~mm}$ $\pm 2.4 \mathrm{~mm}$ lateral to the tip of the coccyx. The total distance between the greater trochanter and the tip of the coccyx was $27.3 \mathrm{~mm} \pm 4.0 \mathrm{~mm}$.

Conclusion: Our results provide anatomical evidence that the ideal needle insertion point is approximately halfway between the greater trochanter and the tip of the coccyx. These guidelines correspond to the technique described for older children and adults.

Key words: Pediatric anesthesia, neonatal surgery, pain management 


\section{Introduction}

Clinical anatomy is linked to good patient care since proper clinical examinations rely on thorough knowledge of gross anatomy [1]. Knowledge of underlying structures helps anesthesiologists to insert the needle towards the target nerve with a measure of confidence resulting in a successful nerve block. The needle can be accurately placed with ultrasound guidance or by using a landmark based technique. Landmark based techniques are often used in Third World or developing countries when ultrasound is unavailable [1]. Whilst technology may facilitate clinical procedures, clinical anatomy remains a cornerstone of medical education and clinical practice [2]. Peripheral nerve blocks of the lower limb are underused in children due to inadequate knowledge of child anatomy [3, 4]. This may explain why Bosenberg et al. found that roughly $80 \%$ of anesthesiologists in developing countries were unable to provide adequate anesthesia for children under the age of five years [5].

The sacral plexus is formed by the fusion of the anterior rami of the fourth and fifth lumbar nerves and the first three sacral nerves [6]. These nerves unite to form the sciatic nerve, which exits the greater sciatic foramen inferior to the piriformis muscle and is covered by the gluteus maximus muscle [7]. In the gluteal region, the sciatic nerve crosses over the superior gemellus muscle, obturator internus muscle and the inferior gemellus muscle and then runs on the adductor magnus muscle as it descends into the posterior compartment of the thigh [8]. The sciatic nerve divides into its two terminal branches, the tibial and common fibular nerves and divides at the posterior aspect of the thigh, proximal to the knee joint. The tibial nerve is the larger of the two branches. It crosses the popliteal fossa superficial and lateral to the popliteal vein and artery, before travelling into the posterior leg [7]. The common fibular nerve is the smaller of the terminal branches; it runs laterally to enter the proximal popliteal fossa along the medial border of the biceps tendon and then curves around the head of the fibular before continuing into the leg $[7,8]$. It has two terminal branches, the superficial fibular nerve, which innervates the lateral compartment of the leg, and the deep fibular nerve, which innervates the anterior compartment of the leg along with some intrinsic muscles of the foot.

A sciatic nerve block is a peripheral nerve block that requires injecting a predetermined amount of local anesthetic solution as close as possible to the nerve without damaging the surrounding structures. A sciatic nerve block provides safe and effective intraoperative and prolonged 
postoperative pain relief after major knee, leg and foot surgery [6]. Complete anesthesia of the posterior aspect of the thigh, hamstring muscles, along with parts of the hip and knee joints and the entire lower leg, except for the medial strip of skin supplied by the saphenous nerve, can be obtained by performing a sciatic nerve block [3]. This makes it a more effective, regional, pain management method than other, more common techniques such as opioid use, which only focus on central sensitization and creates pronociceptive effects [9]. Sciatic nerve blocks can also be used for intraoperative pain relief, as it will interrupt the pain signals before surgery and this can decrease the amount of postoperative pain perceived [10].

Several techniques have been described for performing sciatic nerve blocks at the gluteal level. These include the anterior, lateral and posterior approaches. The anterior approach uses the greater trochanter of the femur and the inguinal ligament as landmarks. A line is drawn parallel to the inguinal ligament and passes over the greater trochanter of the femur and a second line is then drawn parallel to the midline of the body and passes through the junction of the medial one third with the lateral two thirds of the inguinal ligament [9]. The needle insertion site is where these two lines intersect. This method is more painful and difficult to perform and is only used on patients where the other two approaches have been contra-indicated [4]. In the lateral approach the patient is placed in the supine position and has only one bony landmark, the greater trochanter of the femur. The needle is inserted 1 to $2 \mathrm{~cm}$ below the most lateral skin projection of the greater trochanter until a foot twitch - from using nerve stimulation - is elicited [8].

Finally, the posterior approach of Labat was developed on adults and has since been modified for use in children [11]. This modified technique is the most popular because it relies on easily identifiable bony landmarks. The posterior approach is more reliable than the lateral and anterior approaches [9, 12]. In the modified technique, the child is placed in the lateral decubitus position (patient lying on nonoperative side, leaning slightly forward) with the operative leg flexed at the knee and hip; a line is drawn between the greater trochanter of the femur and the tip of the coccyx with the midpoint of that line being the needle insertion site [9]. This technique requires less local anesthetic than other approaches because the overlying muscles envelop the nerve [13]. The procedure is more painful than the anterior and lateral procedures due to the needle moving through the muscle to reach the 
nerve [14]. Nerve blocks at the gluteal level will paralyze the hamstring muscles, resulting in the patient being unable to walk, although in neonates this is not a major concern.

Nerve blocks are usually performed under general anesthesia or under light sedation, to help children cope with their fear of needles. Lightly sedated children cannot indicate if they are experiencing pain associated with paresthesia or neural puncture which may affect success of the block $[7,13]$. Using well-defined landmarks based on accurate anatomical measurements will make nerve block techniques easier to perform and increase block success $[15,16]$. Ultrasound techniques also rely on accurate anatomical interpretation. Our study concentrates on the clinical anatomy knowledge needed to perform a safe and successful sciatic nerve block at the gluteal level in a neonatal population with or without ultrasound guidance.

\section{Materials and Methods}

The sciatic nerve in the gluteal and thigh regions of 20 , formalin-fixed, neonatal cadavers was carefully exposed on the left and right sides. The cadavers of donated neonates of very low to normal birth weight, between the ages of 0-28 days old were obtained from the Department of Anatomy, University of Pretoria. The length and weight of each neonatal cadaver was recorded. Neonatal cadavers were excluded if they were visibly damaged or had abnormalities in the thigh and leg region. Ethical clearance was obtained from the Research Ethics Committee at the University of Pretoria (224/2015) and all dissections were done under the rules and regulations of the South African National Health Act, 61 of 2003.

Due to the embalming process of the neonatal cadavers, they could not be placed in the lateral decubitus position described above. Thus, each neonatal cadaver was placed in the prone position with both legs straight and resting on the table. The skin covering the gluteal region was reflected laterally in order to expose the gluteus maximus muscle which was then reflected from its insertion point on the proximal femur. The fascia was carefully removed, exposing the underlying structures. The following bony landmarks were palpated and marked with colored pins, the greater trochanter of the femur (point A) and the tip of the coccyx (point B). Finally, the sciatic nerve was identified where it 
exits inferior to the piriformis muscle; it was cleaned and the midpoint of the nerve was marked with a colored pin (point C) (Figure 1).

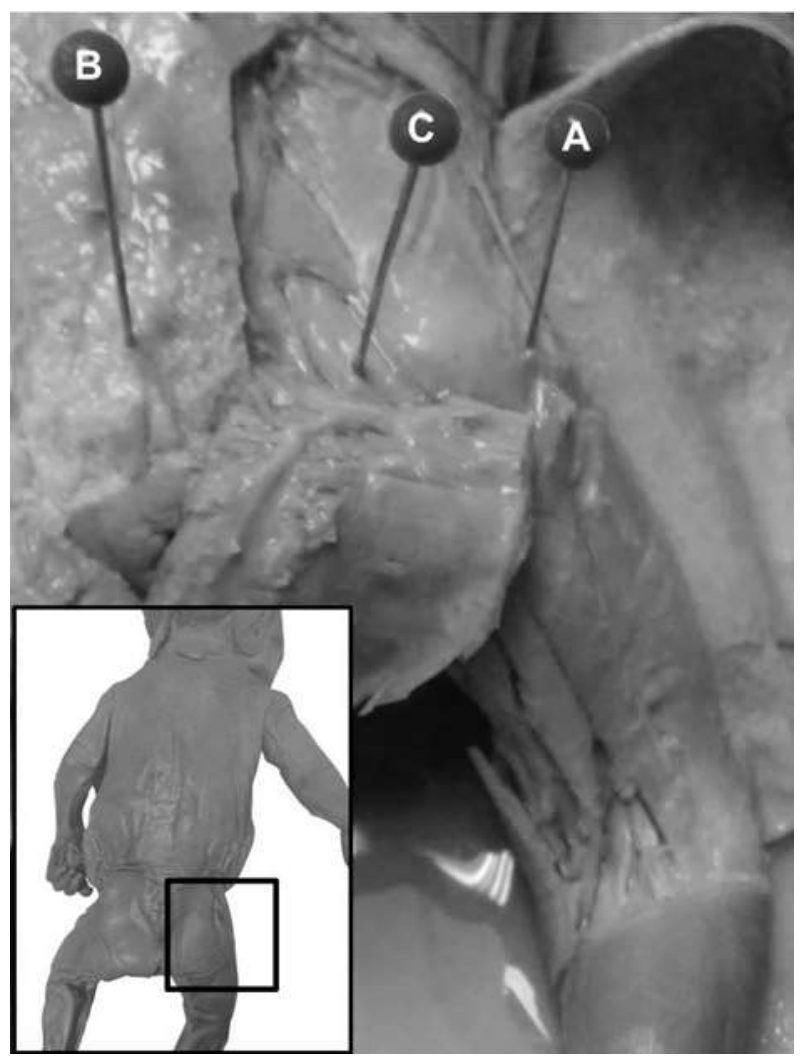

Figure 1: Anatomical landmarks measured after dissection of neonatal cadavers. A: the greater trochanter of the femur, B: the tip of the coccyx and C: the midpoint of the sciatic nerve.

We measured the distance from point $A$ to point $C$, point $B$ to point $C$ and finally from point $A$ to point $B$ on the left and right sides of each cadaver. The distances were measured with a mechanical dial caliper (accuracy: $0.01 \mathrm{~mm}$ ) and recorded. The location of the sciatic nerve in the gluteal region was quantitatively assessed using descriptive statistics. The measurements of the left and right sides were compared using a paired t-test. We also tested for a relationship between collected measurements (dependent variable) and the length and weight of the neonatal sample (independent variable) using a Pearson's correlation test. A r-value between 0.75 and 1.0 will be considered a high (strong) correlation between the independent variables and the measure distance(s), a moderate correlation will have a r-value between 0.5 and 0.74 , while any $r$-value less than 0.5 will be considered as a poor correlation. Measurements were captured in a MS Excel worksheet and Statistix software (ver. 8) was used for statistical analyses. 


\section{Results}

There were no statistically significant differences between left and right measurements (paired t-test, $p$-value $>0.05$ ). Lateral measurements were combined to form a total sample size of $n=40$. There was a poor correlation between the distance of the sciatic nerve to the greater trochanter and the height and weight of the population ( $r=0.2647$ and $r=0.1056$, respectively). There was also a poor correlation between the distance from the tip of the coccyx to the sciatic nerve and the height and weight of the population ( $r=0.2895$ and $r=0.0070$, respectively).

Table 1 summarizes the results obtained for the measurements in the gluteal region. The mean distance between the greater trochanter of the femur and the midpoint of the sciatic nerve was 12.1 mm (95\% confidence interval=11.4-12.7) (Figure 1, Table 1). The mean distance between the tip of the coccyx and the midpoint of the sciatic nerve was $14.9 \mathrm{~mm}(95 \%$ confidence interval=14.2-15.7) (Figure 1, Table 1). The mean distance between the greater trochanter of the femur and the tip of the coccyx was $27.3 \mathrm{~mm}$ (95\% confidence interval=26.0-12.7) (Figure 1, Table 1).

Table 1: Measurements in the gluteal region of the neonatal cadavers (combined $n=40$ ). $\quad A=$ the greater trochanter of the femur, $B=$ the tip of the coccyx and $C=$ the midpoint of the sciatic nerve.

\begin{tabular}{lccccc}
\hline & Weight $(\mathrm{kg})$ & Height $(\mathrm{m})$ & $\mathrm{A}-\mathrm{C}(\mathrm{mm})$ & $\mathrm{B}-\mathrm{C}(\mathrm{mm})$ & $\mathrm{A}-\mathrm{B}(\mathrm{mm})$ \\
\hline Mean & 1.0 & 0.5 & 12.1 & 14.9 & 27.3 \\
Standard Deviation & 0.9 & 0.2 & 2.1 & 2.4 & 4.0 \\
Minimum & 0.3 & 0.3 & 6.4 & 9.7 & 16.6 \\
Maximum & 4.0 & 1.1 & 15.0 & 22.0 & 34.7 \\
Range & & & $11.4-12.7$ & $14.2-15.7$ & $26.0-28.5$ \\
$(95 \%$ Confidence Interval) & & & & & \\
\hline
\end{tabular}

\section{Discussion}

We describe the clinical anatomy of neonatal cadavers without leg or hip deformities. We reveal that little anatomical variation exists in neonatal cadavers, making the greater trochanter of the femur and tip of the coccyx suitable landmarks for conducting sciatic nerve block without ultrasound guidance. 
In the gluteal region of neonates, the mean distance from the greater trochanter of the femur to the tip of the coccyx was $27.3 \mathrm{~mm}$ and mean distance from the tip of coccyx to the sciatic nerve was 14.9 $\mathrm{mm}$. The optimum needle insertion point when performing a sciatic nerve block in neonates based on the findings in this study is midway between the greater trochanter of the femur and the tip of the coccyx. Our findings corroborate with the recommendations made by Dalens [10] and Ross et al. [12].

Placements based on landmarks allows for individual anatomic variation arising from ontogeny. We located the sciatic nerve of neonates just outside the midway point between the greater trochanter of the femur and the coccyx. The nerve was marginally closer to the greater trochanter. In the largest neonate examined, the nerve was located $22 \mathrm{~mm}$ from the tip of the coccyx where the total distance was $34.7 \mathrm{~mm}$-placing the sciatic nerve $4.7 \mathrm{~mm}$ lateral to the midpoint between the greater trochanter and the coccyx. Placing the needle at the midway point $[10,12]$ in this case may have had a chance of missing the nerve. We thus recommend that nerve stimulation be used to confirm accurate placement (eliciting knee flexion, adduction of the ankle, or plantar flexion of the hallucis) when performing sciatic nerve blocks without ultrasound guidance. In contrast to what has been reported in other studies [17], the authors did not observe any high division of the sciatic nerve, especially not in the gluteal region. The highest point at which the sciatic nerve is divided into the tibial and common fibular nerves was in the mid-thigh region, and is thus unlikely to influence a success of a block when the needle is placed in the subgluteal region.

There are limitations to this study. The measurements were made on formalin-fixed, neonatal cadavers. There is a possibility that the embalming technique could alter the underlying anatomy and potentially cause shrinkage or anatomical distortions. These distortions are negligible as the anatomy seen in a cadaveric sample is similar to living studies [16]. The cadavers used in this study were preterm or full-term neonates who had died from perinatal complications. Most of the cadavers were thus of extremely low or low birth weight neonates with an average weight of $1.2 \mathrm{~kg}$. This also meant that the standard deviation was high as the weights varied within the sample. The anatomy of infant patients is closer to the anatomy of a neonatal sample—used in this study —-than to that of an adult sample and we suggest that extrapolating data obtained from neonates is more appropriate for infants and young children than those gathered from adult studies 
In conclusion, we have shown that the sciatic nerve is approximately halfway between the greater trochanter of the femur and the tip of the coccyx in neonates. Our measurements concur with other published recommendations $[10,12]$ and we validate the posterior approach for performing sciatic nerve blocks at the gluteal level. We suggest that in neonates, the distance between the greater trochanter of the femur and the sciatic nerve is too variable to justify using the lateral approach without ultrasound guidance.

The use of landmark-based techniques is still relevant in regional anesthesia despite the increasing use of ultrasound-guided nerve blocks. These results could be used to guide ultrasound probe placement when ultrasound is available in neonates and infants.

\section{Funding}

The study received no external funding.

\section{Disclosures}

The authors mention that Prof. AT Bösenberg (coauthor) is a Section Editor for Pediatric Anesthesia. This project was approved by the Research Ethics Committee at the Department of Anatomy, University of Pretoria (224/2015). The costs of this project were covered by the Department of Anatomy, University of Pretoria. The authors also thank the National Research FoundationCompetitive Support for Unrated Researchers (NRF-CSUR) Grant for their financial assistance. Any opinion, finding, and conclusion or recommendation expressed in this material is that of the author(s), the NRF do not accept any liability in this regard. The authors acknowledge Dr Cheryl Tosh of the research office for her language editing as well as the families of, and the body donors themselves without whom this research would not have been possible. 


\section{References}

1. McCuskey RS, Carmichael SW, Kirch DG. The importance of anatomy in health professions education and the shortage of qualified educators. Acad Med. 2005 Apr 1; 80 (4): 349-51.

2. Miles KA. Diagnostic imaging in undergraduate medical education: an expanding role. Clin Rad. 2005 Jul 31; 60 (7): 742-5.

3. Dalens B [Internet]. Lower extremity nerve blocks in paediatric patients. NYSORA; [updated 2013 Oct 8; cited 2015 Sept 15]. Available from: http://www.nysora.com/.

4. Oberndorfer $\mathrm{U}$, Marhofer $\mathrm{P}$, Bösenberg A, Willschke H, Felfernig M, Weintraud M, Kapral S, Kettner SC. Ultrasonographic guidance for sciatic and femoral nerve blocks in children. $B J A$. 2007 Jun 1; 98 (6): 797-801.

5. Bösenberg AT. Pediatric anesthesia in developing countries. Curr Opin Anaesthesiol. 2007 Jun 1; 20 (3): 204-10.

6. Dadure C, Capdevila X. Continuous peripheral nerve blocks in children. Best Pract Res Clin anaesthesiol. 2005 Jun 30; 19 (2): 309-21.

7. Ellis $\mathrm{H}$, Mahadevan V. Clinical anatomy: applied anatomy for students and junior doctors. John Wiley \& Sons; 2013 Aug 13.

8. Faiz O, Moffat DB. Anatomy at a glance. Blackwell Pub; 2002

9. Dalens B. Lower extremity nerve blocks in pediatric patients. Tech Reg Anesth Pain Manag. 2003 Jan $31 ; 7$ (1) :32-47.

10. Hunt KJ, Bourne MH, Mariani EM. Single-injection femoral and sciatic nerve blocks for pain control after total knee arthroplasty. J Arthroplasty. 2009 Jun 30; 24 (4): 533-8.

11. Labat G. Regional anesthesia: its technic and clinical application. WB Saunders; 1922.

12. Ross AK, Eck JB, Tobias JD. Pediatric regional anesthesia: beyond the caudal. Anesth Analg. 2000 Jul 1; 91 (1): 16-26.

13. van Geffen GJ, Pirotte T, Gielen MJ, Scheffer G, Bruhn J. Ultrasound-guided proximal and distal sciatic nerve blocks in children. J Clin Anesth. 2010 Jun 30; 22 (4): 241-5.

14. di Benedetto P, Bertini L, Casati A, Borghi B, Albertin A, Fanelli G. A new posterior approach to the sciatic nerve block: a prospective, randomized comparison with the classic posterior approach. Anesth Analg. 2001 Oct 1; 93 (4): 1040-4. 
15. Reinoso-Barbero F, Saavedra B, Segura-Grau E, Llamas A. Anatomical comparison of sciatic nerves between adults and newborns: clinical implications for ultrasound guided block. $J$ Anat. 2014 Feb 1; 224 (2): 108-12.

16. Bosenberg AT. Regional anaesthesia in children: an update. South Afr J Anaesth Analg. 2013 Jan 1; 19 (6): 282-8.

17. Gehdoo RP. Post-operative pain management in paediatric patients. Indian J Anaesth, 2004; 48: 406-414.

18. Loepke AW, Soriano SG. An assessment of the effects of general anesthetics on developing brain structure and neurocognitive function. Anesth Analg. 2008 Jun 1; 106 (6): 1681-707. 